\title{
Interview
}

\section{An interview with Henrik de Gyor, K12 Inc.}

\section{Henrik de Gyor}

is the Digital Asset Manager at K12 Inc. Henrik manages the daily operations, the future expansion and the implementations of the digital asset management system. Before being at K12, Henrik was a staff photographer and photo editor working on newspapers, magazines and annual reports in Washington DC area. He was an adjunct professor teaching core classes at the Corcoran College of Art and Design for 3 years. In his spare time, Henrik blogs on a regular basis about DAM in the user and the administrator perspective at http://www.anotherdamblog.com.

\section{ABSTRACT An interview with the Digital Asset Manager of K12 Inc., Henrik de Gyor, on the implementation and daily operations of the Digital Asset Management within the education company.}

Journal of Digital Asset Management (2010) 6, 173-181. doi:10.1057/dam.2010.15

Keywords: Henrik de Gyor; K12; Digital Asset Manager; education; DAM; Digital Asset Management

Correspondence: Henrik de Gyor K12 Inc. 2300 Corporate Park Drive, Suite 200 Herndon, Virginia 20171 E-mail: hdegyor@k12.com

\section{ABOUT K12 INC.}

K12 Inc. (NYSE: LRN), a technology-based education company, is the largest provider of proprietary curriculum and online education programs for students in kindergarten through high school in the United States. K12 provides its high quality, award-winning curriculum and academic services to online schools, traditional classrooms, blended school programs, and directly to families.

In partnership with charter schools and school districts, K12 operates online public schools in 25 states and the District of Columbia. K12 Inc. also operates the K12 International Academy, an accredited, diplomagranting online private school serving students in over 40 countries. Since its founding, K12 has provided more than 1.5 million courses to over 150,000 students worldwide. More information can be found at www.K12.com. MM: We are here with Henrik de Gyor of K12 Inc. Henrik, why do not we start off with a little bit of your background, current and a little bit of your professional background as well.

HG: I am the Digital Asset Manager at K12 Inc. Essentially, I manage the daily operations, the future expansion and the implementations of the digital asset management system. The DAM holds our audio, video, text, graphics and photographs, which K12 uses to build our curriculum from scratch. We also have expanded to incorporate not only our product development, which creates our curriculum, but also our marketing department, operations and our subsidiaries. Prior to being at K12, I was a staff photographer and photo editor working on newspapers, magazines and annual reports in Washington DC area. I was an adjunct professor teaching core classes at the Corcoran College of Art and Design for 3 years. In my spare time, I blog on a weekly basis about DAM in the user and administrator perspective at http://www. anotherdamblog.com.

MM: It sounds like a great place to launch into kind of a deep dive interview in DAM. Now, as I understand it Henrik, you were part of the team that specified and/or researched the requirements for DAM.

HG: Yes.

MM: Could you just take us through kind of what was going on at K12 and specifically what happened or what precipitated the management's interest in deploying them. HG: Sure. K12 needed a way to search, find, use and reuse photographs specifically and a 
way to track the rights of those photographs, because most of the photographs were acquired from outside vendors. Once we investigated the fact that we needed digital asset management to do that, and we identified a few vendors that would be able to do this, we took it a step further and said well we are accumulating a lot of digital assets nowadays. Why not do this not only for photographs, but also for text, graphics, audio and video, and be able to find all those.

MM: Right and as you opened up that larger can of worms, so to speak, of all these different kinds of things that you keep track of, these items both in their master state as well as derivatives and renditions, these are all elements of core products that K12 makes.

HG: Yes.

MM: So, what consideration did you put in to not just a place to find things, but also to support, say, the core business process of creating curriculum and selling it?

HG: We identified the fact that if we were not able to find those assets, we would have to reacquire them if we were just acquiring outside assets, but a large portion of our curriculum is created in-house where we create assets from scratch, and if we can't find them again, then we have to spend more time recreating them. In order to keep those master files per se as well as the derivatives, if you want to call them master files or parents, and derivatives' children, we keep both of them as often as possible, and we are able to reuse any of them; if we already have a child that fits the needs, we can reuse that or we can repurpose any of them when we know we are permitted to do so.

MM: Sure. About how many files are we talking about at this point?

HG: [At the time of this interview], we have about 100,000 assets.

MM: What did you have prior to the DAM was there a sense as far as how much external purchases were entailing in terms of cost and/or if not cost the number of times that you were outside to buy things that are not functionally having a DAM?

HG: Yes, we were identifying the fact that every time we could not find something we would repurchase it again. That was obviously a loss that we wanted to curb as quickly as possible.
MM: Any sense of magnitude in terms of how often that was happening?

HG: Too often. Probably every project encountered that issue.

MM: And typical procurement cost of these things, were these kind of rights-managed photos or were these royalty-free kind of photos?

HG: Both, and so, there is a liability issue with the rights-managed photographs that we wanted to limit as much as possible. We had some tracking methods involving spreadsheets, which were to a degree archaic that we needed to ameliorate. We decided that the DAM should be able to do this in order to centralize the assets along with tracking the rights to use them. That was quickly found to be a bit of a challenge since no Digital Asset Management System we could find did this, and we looked at 90 DAM vendors. We had to customize the DAM in order to do this.

MM: About how many royalty-managed images are you talking about having to manage?

HG: I am sorry, royalty free or rights managed? MM: Rights managed, sorry.

HG: Oh, rights managed, I would say between the royalty free and the rights managed, it is a 3:1 ratio. There is about one rights-managed photograph for every three royalty-free photos.

MM: And so, we are talking what 10,000 or so all together or ...

HG: More like in the range of 40,000 .

MM: Yeah, so that represents a fairly significant risk that you wanted to mitigate.

HG: Yes.

MM: So, as you looked at these 90 or so systems, were you part of the team that looked at these 90 systems?

HG: Yes, I was basically the person looking at every single one and evaluating them specifically for our business needs. Some did not meet certain criteria which we had, some had stronger features than others and some would require more customization than others to meet those needs, so many were vetted. We looked at cost, the features they had and how they were designed to handle different workflows such as uploading, for example, which varies quite a bit. We even looked at the customer service level as far as how many technical, client-facing customer service people could answer our question if we were on a deadline 
on a Friday at 5:00 p.m. Who would answer our call and answer our questions then? That basically weeded out a lot of the vendors.

MM: So, as you kind of went from 90 down to a short list of you know 10 or 20 or however many it came to, what criteria did you use in selecting the really short list or the final candidate?

HG: We looked at company history. Have they existed for a while? Is it financial sound? Have other organizations such as education companies used them? Do they have a good track record? Again, what features do they have 'out of the box' and what options are missing that would need to be customized. Again, their rightsmanagement component was something that pretty much no one had, not to the robust quality or ability that we would need to track assets.

MM: That is something that you subsequently built?

HG: Yes, we had to customize that from the ground up and we picked a vendor that had the beginnings of it, and we basically dissected the products to see well if you can already do this part, can you add these components on top of it, customize it and meet our particular business needs. We created a statement of work that was very involved once we picked the product that we needed. One of the challenges that we had was to identify what is the level of configuration available in the product and where does that end? Where do we need to add a customization in order to meet our needs? That is something we struggled with for a while until we documented everything that we needed.

MM: And in the course of doing that, can you say about how long it took to develop your statement of work?

HG: The statement of work probably took a few months to develop in its entirety and then of course the building of it and the testing of it took a bit longer.

MM: Sure, I am really curious in terms of this front-end work in terms of really understanding your requirements and then expressing as a statement of work. You say that that really came after you had more or less set upon your final candidate. Is that correct?

HG: Yes.

MM: And so at that point, had you yet purchased the system or did that then become the basis for them putting together your statement of work that ultimately led to the purchase.

HG: At that point, we had already licensed the product.

MM: Okay, so you were in the metaphor of the chicken and the egg, the chicken and the pig at breakfast, you were more or less the pig. You were committed to the ham.

HG: To a degree. We were committed for some time and we wanted to make sure when the renewal come up that this was still the vendor we wanted and it came about that it was.

MM: So, in that context then in putting together the statement of work to what extent did you get into, how can I say explicit models of workflow and how detailed did you get that? HG: We went through the entire workflow as far as the system sought from the time you would enter the system to the time you would exit with an asset or simply exit the system after uploading assets with metadata.

MM: How did you do this, did you do this with wire frames or marked up screen shots or swim lane diagrams.

HG: We used some of those and to a degree we marked up some screen shots, but for the most part, it was built from scratch because there was no tool out there that did what we needed to. So, we came up with what all the criteria necessary to meet those needs, particularly for the rights-management component.

MM: And at this point this also then probably entailed developing some pretty well-defining use case models, was that.

HG: Yes. We had multiple workflows.

MM: Yeah, and so could you take us through how you develop those use case workflow or your use case models, which were adapted to the workflow and to what extent did you involve particular users in the development of those used cases?

HG: From the very beginning, we interviewed users or potential users even prior to even acquiring the DAM to model exactly what their needs and wants were, even if they did not know what was available. While we may have had a glimpse at the time to what DAM could do for us, but many people are not used to DAM nor what it could do for them. We said 
where the gaps are and what could be fixed if you had a tool that could do this or that or the other thing? How do you search currently without a DAM? Those responses were compiled, documented and essentially rebuilt with models based on roles, so we had different roles within our organization and that remains the fact today where we permission by role. If a particular role needs to do certain things, which we identified in the past, they would be given those specific permissions to see certain assets and do certain tasks with those assets. That worked quite well.

MM: And, how did you develop your metadata and taxonomy framework?

HG: Great question. Because we were involving photographs, text, graphics, audio and video, we did not want multiple metadata schemas. We wanted to make it as encapsulating as possible. We were able to come up with actually two models that covered all of those components including the parent and the child assets of all those assets. There were differences between a few types of assets that were quite numerous. That was the only reason we needed another one of those metadata schemas but they were mostly mirrored with each other.

MM: Henrik, would that classically be represented as facet within a taxonomy or you are talking about two kind of fairly complete taxonomies that are complementary, but also reflect to fairly, how can I say, comprehensive ways of looking at all of the assets.

HG: Yes. It worked well because we basically went through examples and models of every kind of asset that we had currently, and what we were expecting to have in the near future. We knew what we would definitely need as required metadata fields so we could not upload an asset without having that field filled in with specific metadata. Often we were able to use drop-down values with real preset values and some of them were free text. We tried to limit the free text fields as much as possible, but we knew that that was a necessity to have that free text in order to identify some factors.

MM: And as it relates to then, about how many metadata field values you typically associate with an asset?

HG: We have a total of 40 fields and we have about 15 that are required.
MM: And, who principally is responsible for putting the metadata into the system?

HG: Great question. It depends on whether we acquire the asset from an external vendor where one particular role, which we define as the media editor, actually acquires it and licenses it. If it is acquired from an external vendor, they bring in the metadata from that vendor, they normalize the information and they uploaded it with the asset. If it is created by us, granted we are the only ones who know about the asset, so no one else can tag it without institutional knowledge in a timely manner, we have the person who requests the asset that while they are requesting it, they create metatags for it. After it is created, we have a series of individuals who we define as information specialists, who upload those assets with the associated metadata.

MM: And, that is also where the normalizing function takes place?

HG: Yes. They also normalize it there.

MM: Cool, could you take us a little deeper into the domain of rights management and some of the issues that you had to work through in developing that as an application.

HG: Sure, we looked at several models out there. I looked at PLUS, PRISM and several other ways to manage rights, but unfortunately they were not robust enough for our particular needs. Now, granted those standards are quite well developed, but not to our business case which tracks assets so the usage is clearly defined without using proprietary codes. MM: Can you give me a couple of examples of reports that you needed to satisfy, but you could not on those existing approaches.

HG: Sure. For example, we have third-party rights. If we have a Picasso in a photograph, which we get from a particular vendor, not only do we have to license the photograph, but we have the third-party vendor, which licenses it from the estate of Picasso that we need. So, we have the image itself that we receive from a vendor and then we have to license it from another third party, which adds to the cost and crediting necessary in order to use it. We did not see a model that sufficed for that and we also did not see a model that permitted reuse of assets. Now, granted you cannot really reuse an asset that is rights managed unless you have another license per se, but we wanted to avoid 
having multiple copies of the same asset. We wanted one asset with multiple licenses for it. This avoids the 'Oh, here is a PDF attachment of the license that we get from the vendor. Read on each time'. We actually wanted to spell out the license terms in component form, so that we knew we could export reports and we could actually figure out how much we were spending per vendor as well as per project. MM: Yeah and so many of these contracts or licenses may entail a fairly lengthy contract about 10, 15, 20 pages.

HG: Usually not.

MM: About how many pages are we talking about?

HG: Usually one or two.

MM: Oh, really, okay.

HG: Yeah.

MM: And, so you are saying that instead of simply rendering a PDF of that particular license, you would want to break it apart, basically extract the text and put it into the DAM system as metadata or at least, anyway, information attached to the asset.

HG: Yes.

MM: Cool. Okay and about how many licenses are we talking about having to manage?

HG: Well, we have a license or agreement for all the images we use.

MM: 20,000 or 30,000 or something like that.

HG: Actually, probably more. Granted, we start tracking licenses with the DAM after the asset is uploaded into the DAM. We are also going back and doing renewals for licenses, since there is a term and expiration for all those rights-managed licenses. So, as we renew them, we license them and add that license in the DAM. We are seeing many thousands of licenses.

MM: And so did at any one point in time was there a conversation around using a Document Management System to manage those as documents or did you simply want to have them all brought into the DAM.

HG: Well, the idea behind the DAM is we wanted to essentially enable, unify and simplify the process as much as possible. We have a Document Management System, but we use it to manage other documents, not contracts. We do not manage contracts per se in that Document Management System, because you have to discern between one contract and the other. After reading, it is not logical to have all those contracts in a Document Management System where you have to re-read each one of those contracts in order to figure out what your rights are and can you reuse this, how much will it cost for you to reuse this in a timely fashion. I know some organizations do this, but I do not see that as a logical way if you cannot report on it in an instant fashion, on the asset level.

MM: So you really hit upon, I think, a key dimension not well understood of the DAM system and that is unified reporting and reporting not just on who is using what or who downloaded what, but other things as relates to managing the asset such as are we in compliance, is it up for renewal and so on. Could you just take us, give us a quick primer of reporting and the robust DAM, kind of highlighting the various business functions and the kinds of reports that you are able to produce in this kind of more mature DAM implementation of yours?

HG: I create reports on a monthly basis, but we can create reports anytime we need. I create a monthly reuse rate where we look at every aspect and how many times we have actually used an asset to see well how much return on investment have we gotten for these assets. I also run weekly reports on how many active users we have in the DAM as well as how many assets we have, and categorize them by the type of assets.

MM: Do you actually calculate aside from, you know, count rights, $N$ number of assets were used $Y$ number of times? You actually estimate or guesstimate the economic valve of an instance overuse or a class overuse.

HG: Yes, because we know how long it takes to create an asset and we know exactly how much it costs to acquire that asset. When we acquire that asset, we know we spent $X$ and we know however many times we used it.

Otherwise you would may have spent $X$, but if you did not find it again or reuse it again, there is little ROI on the asset level. The same applies if we created the asset internally, if we spend $X$ number of hours creating the asset, you want to avoid spending the same amount of time to recreate that same exact asset. We measure the amount of use assets get within projects as well as across projects. 
MM: So in your model then you have the various roles with an hourly rate. Hopefully, it is a fully burdened hourly rate in terms of their salary overhead and all that kind of stuff right.

HG: Yes, we can.

MM: And then you look at how much time the system saves that particular role, times the number of instances that that particular user/role affected an activity, etc.

HG: We could do that.

MM: And so in terms of your report to management, do you provide an income statement or some sort of a financial report in terms of the economic performance of these assets.

HG: We work with project managers and finance to determine all those costs and while they know how much each person is making on an hourly basis, even if their salary, yes I supply them with the number of assets, time metrics involved and they can type the cost to those numbers to actually determine the actual cost benefit.

MM: So the actual calculating the return on these assets is done by your project team or your finance team, it is not necessarily done within the DAM operation, is that what you are saying?

HG: Yes. Aside from actually what we spend on the asset itself, no, we do not tie our timesheet software to our DAM.

MM: Sure, now in terms of the economic value of these assets, is K12 a publicly helped firm or is it private?

HG: We are a public company. We are traded on the New York Stock Exchange under the symbol LRN.

MM: Yeah. So in terms of your reporting system what are some of the more interesting things that you have sent out in the data?

HG: We reuse more than we originally thought we would ever reuse. Usage (and reuse) numbers are higher than the total number of assets in the DAM.

MM: Without getting into the specific numbers, can you give me a sense of percentage on that? HG: Percentage-wise. I would say on average we, within a project, can reuse 30 per cent of the assets that we have acquired in the past.

MM: Sure. As we think about DAM operations and being a manager or a director of DAM operations, could you take us through, if you will, the major areas of accountability or concern with respect to the ongoing care and feeding of a DAM system or DAM operations group?

HG: Sure. On a daily basis, I support the project teams' needs, resolve issues and document future needs of the DAM. When it comes to asset use, I generate the reports directly from the DAM to account on every aspect of the usage and metadata. I support and train every user, including power users. I deal with all the issues that are involving with the DAM, from the licensing to future customizations.

MM: And with respect to the kind of control and/or governance associated with roles, policies and metadata, is that also something that falls within your preview.

HG: Yes. I enforce the metadata models that are established, so if someone is giving us garbage metadata, we throw it back and we say 'sorry, this is not acceptable. You need to tag it appropriately based on the following documentation'. One thing that we did instead of supplying the documentation from the vendor directly, we actually rewrote it. Not on paper form. Not in PDF form, but rather on an internet wiki, similar to wikipedia but within our intranet, so that anyone in the company can access the documentation, step-by-step instructions on everything relating to DAM. From the workflow on to how to upload an asset, how to metatag an asset, how to order an asset or how to download an asset, all that is documented both in a user, power user and even admin perspective. If anything would happen to me, for example, it is all there.

MM: So that is great. Could you take us a little more through, you say you developed kind of an interactive user manual support system that essentially has documented everything that you know about the current feeding of the system. How is it organized in terms of is it organized by role or workflow or business process again, just kind of feedback covers of that a little more.

HG: Sure. It took me a few months to write it because I was going through the 800 pages of manuals and guides that the vendor supplied and then added information from our consultation as well as our workflow to it. So as I look at it, it 
appears like web pages that have a Google-type search bar as well as a hierarchical table of contents.

MM: So this is part of an overall organization business policy for having a kind of a documented firm.

HG: Yes, everything is documented. We have a DAM manual, DAM upload guide, upload templates for bulk uploading with metadata, even template questionnaire for new projects. We documented DAM utilities and a DAM admin section, if you have admin permissions, you have access specific functionality. As we go into the DAM manual, we describe everything from what is the Digital Asset Management system, how do we access the DAM, what would we be able to do in the DAM system based on role, how do we use these manuals in case they have not used wiki before, because essentially it is a powerful website with very useful content about DAM. It is almost like a wikipedia where there is a free text Google-like search bar where you can type in what you are searching for and you will find it or you can click on the links supplied and it will lead you to the necessary page, same thing as FAQ page where it is just a list of questions that are typically asked and if I get a user question, I will forward them a URL to that question/answer in the wiki. I do not have to retype an e-mail repeatedly to answer the same question.

MM: So you say it took several months to write this wiki type of user support.

HG: Yes, I mean if you were to print it out it will be about 100 pages plus all the links that were made to it and, granted, if anything changes I can change it instantly and it is viewable to everyone, so not only is the change viewable to everyone but everyone who signs up for the daily e-mail from the system get a rundown of every change that occurs on a per page basis and on a per link basis. If they see that particular favorite page changed they can click on that and say 'Looks like that workflow changed or this policy changed or this is how we should handle it now instead of how we did it last month'. I find well worthwhile.

MM: Yeah. Okay, cool. And in terms of kind of forward look on ideas in terms of where you see this evolving, how do you see your particular DAM evolving?
HG: I regularly look at different parts of the organization to see how DAM can help them, because I do not believe in the model of having multiple DAM systems within an organization or having a DAM per department, because there is the option of having permissions for collection if you will, where one department can only see these sets of assets and the other departments can only see those sets of assets and then some departments can see any asset, so I regularly look at different organizations and hear about different needs.

MM: How about integration with other systems such as your content management, your document management or financial systems? HG: So, our DAM is tied to our Content Management System (CMS). It feeds assets to it on a regular basis and vice versa. As we develop our CMS even further to meet further business needs, we have additional requirements for the DAM that we will have to evaluate and build on.

MM: And does the K12 also have a learning management or an LMS system.

HG: We have multiple. And because we have multiple, we need the flexibility to deal with those multiple learning management systems. Therefore, our CMS has to be as flexible as possible in order to do that.

MM: Yeah, so then would it be fair to say that, in the future, you would look at having more of a federated DAM space where you have assets that are in your DAM, that are, if you will, in the garden of metadata and policy as well as assets that are outside the DAM; say for example on a learning management or web content management system that what you have is you have a link to and then metadata of a surrounding met link that may give a DAM user the ability identify and perhaps retrieve an asset that lives in one of those systems?

HG: To a degree, we already do that. The idea is that the student should not need the DAM itself nor even the CMS for that matter, but should be able to consume the lessons and the materials we provide for them as well as external links.

MM: I was thinking more not so much from the student's experience of the learning management system, but more from a teacher or a curriculum designer looking at not just the things that are in the DAM system, but things 
that are outside of the DAM system, the search function within the DAM has extended the arm of what is in the repository itself to include assets that are outside it.

HG: Well, I understand what you are saying now. We currently do not do that only because we want to be able to know what we have rather than what we do not have and be able to license it properly, because there are so many restrictions and liability to things that are just available in different places even with established vendors.

MM: Finally, as we are kind of moving forward here into the next phase of your DAM operations, do you anticipate doing any kind of like a user conference or getting together with users or is that something that is already kind of being done on a day-to-day basis, as a function of how you are interacting with them.

HG: We regularly get feedback from users and that is how I am able to support them in all their DAM needs along with project needs rather quickly. We do not have any user conferences. We found instructing users when they have assets to work with and they use the DAM, they tend to yield a higher rate of success instead of watching me go through a workflow. As far as users, I value their opinions and their feedback so that the DAM can be user friendly and satisfy their business needs.

MM: Does that entail doing, say for example, a semi-annual or annual audit/survey of your users?

HG: We have currently not done that and we do plan on awarding them in the near future based on activity, so giving them a DAM award based on the highest activity for that role, because we can identify, 'You were the most active user within this role during this month'. We will probably be awarding people for their reference and their encouragement of others via their peers and managers successfully using the DAM. I report on the number of active users and assets in DAM as part of my weekly report. MM: You know I ran across a handful of companies that actually have a formal rewards system in place where they create a monthly or quarterly award to users that have created the asset with the highest amount of reuse by category, you know photograph, image, video, etc, most innovative expression of the brand, etc. Lot of different ways of again creating incentives. Also, in terms of not just the creators, but the downloaders, using promotions as a way to intent good behavior.

HG: That makes sense. The reporting features of the DAM gives you an objective view of what is being done in the DAM to the degree that you have hard data that can generate numbers to be filtered, yield the productivity figures, the progress of what the organization is doing and the DAM's progress.

MM: One of the things that we ran across in a couple of really large publishing firms especially in the text book area was they created an internal marketplace, where they had multiple design centers and multiple business divisions and from the Chief Learning Officer (CLO) I think it came to mandate that you have to make all of your assets available through all of your sister companies, and as a way of inducing good corporate citizenship, they said that and you will have the right to sell these assets to the subsidiaries for one-tenth of the share market value of the image or illustrations.

HG: To cover the cost I guess.

MM: Well, now in truth it was not a taxable event. It was not, because it was just an internal transfer, so it was kind of an internal accounting system, but they were using real dollars in somebody's Profit \& Loss (P\&L).

HG: Like a charge back system.

MM: Yeah, kind of like a charge back system. Again, the key point was it was not a taxable revenue event, but in terms of the overall P\&L performance of a particular division, it showed up as contributions to their revenue, obviously a charge back mechanism, and this has a net result of putting in place a structural incentive and a top-down mandate, one: to create reusable stuff, two: upload and tag it right, and three: promote it to your sister firms, that is, not hide it, and as a function of this marketplace, they created an internal marketplace, they produced a 19 times return on investment over its first year. HG: That is great.

MM: As it relates to a textbook, they did kind of a pilot across four textbooks looking at the costs and cycle times associated with these five textbooks, and they were able to reduce production costs across these five textbooks by US $\$ 30,000$. They were able to eliminate 
another $\$ 75,000$ of soft costs associated with the reduction of the textbook. They were able to eliminate per text book an average of five traffic meetings, traffic or production meetings with a head on average seven people per meeting, which went into that $\$ 75,000$ savings and they reduced their time to market for new curriculum by 4.5 weeks, so there was tremendous measurable economic benefit in terms of lower external spent, lower labor costs associated with reducing the text book and a faster time to market, which seems to be, if you will, our whole point of kind of our framework around our conversation today is that DAM is more than just a technology or assistance. It is really a business strategy for reducing costs, speeding cycle time and creating a level of transparency where none had really existed before.

HG: And that is vital to the companies today.

MM: Well, I wish you great success.

HG: Thank you.

MM: In developing this thing and you have done several things just absolutely consistent with next practice and best practice, so congratulations.

HG: Thank you.

MM: Any last words that you like to conclude on?

HG: Well, the DAM has been quite successful. Its success has been a long road, but we do not want to sit on our laurels. Instead, we look at how we can improve it. Thank you.

MM: Thanks again. 